\title{
Responses of different phytoelements to habitat light level and their dynamic convergence towards crown development of Aucuba japonica Thunb. var. japonica
}

\author{
Md. Sohrab Ali ${ }^{1, *}$ and Kihachiro Kikuzawa ${ }^{2}$ \\ ${ }^{1}$ Department of Environment, E-16, Agargaon, Dhaka 1207, Bangladesh \\ ${ }^{2}$ Department of Environmental Science, Ishikawa Prefectural University, Suematsu, Nonoichi-cho, Ishikawa 921-8836, Japan
}

\begin{abstract}
We analyzed crown development in Aucuba japonica Thunb. var. japonica resulting from the responses of phytoelements to habitat light conditions over a long period of time. Over the years, the degree of extension unit (EU) dimorphism and the degree of anisophylly were higher under shaded conditions than in brighter conditions. An overall temporally increasing pattern in the degree of EU dimorphism was found while no clear-cut trend was found in the case of anisophylly. EU length and number of leaves per EU co-varied in a spatio-temporal context. The number of terminal buds and their sizes acted as the key initiators of morphological differences of phytoelements which were further amplified following bud break. Leaf area density was displayed mostly in the apex peripheral layer of the crown and the apex layer received most of the incident light. There was a tradeoff between annual leaf production and mean leaf size. Depending on the heterogeneity of irradiance level within a crown, correlative growth inhibition caused higher EU mortality at brighter sites. Due to high mortality, shorter EUs had a mere role in the construction of structural framework of the crown except for the formation of some gaps. There was a strong convergence of EU dimorphism, anisophylly, EU extension growth and variations in leaf size towards formation of functional crown to reduce potential self-shading. Depending on the irradiance level, Aucuba japonica Thunb. var. japonica showed two different modes of crown expansion. At the brighter sites, individual crown expansion was progressive while at the darker sites, individual crown expanded in a diminishing manner and maintained a stable size. A plant's "growth diminishing phase" appeared earlier at shaded sites than brighter sites.
\end{abstract}

Key words: anisophylly, crown formation, EU dimorphism, light response, phyotoelements dynamics

\section{INTRODUCTION}

A leaf receives environmental signals and goes under frequent modifications to develop into an adaptive organ size (Goebel 1900, van Volkenburgh 1999, Yano and Terashima 2004) like anisophylly (Ali and Kikuzawa 2005a). Similarly, extension unit (EU)/stem often modifies itself to develop adaptive functionality for example the difference in EU length (Maillette 1982, Ishihara and Kikuzawa 2004). Longer shoot (combination of EU and leaves) specializes in space acquisition while shorter shoot works as gap fillers (Whitney 1976, Hallé et al. 1978, Jones and Harper 1987, Yagi and Kikuzawa 1999). All the modifications performed by a plant's internal and external resource availability (Kume and Ino 2000, Ishihara and Kikuzawa 2009) accrue to develop a functionally adaptive crown.

Establishment and survival of understory plants de-

\section{Open Access http://dx.doi.org/10.5141/JEFB.2012.021}

This is an Open Access article distributed under the terms of the Creative Commons Attribution Non-Commercial License (http://creativecommons. org/licenses/by-nc/3.0/) which permits unrestricted non-commercial use distribution, and reproduction in any medium, provided the original work is properly cited.
Received 04 February 2012, Accepted 07 June 2012

*Corresponding Author

E-mail: md.sohrab_ali@yahoo.com; sohrab@doe-bd.org Tel: +880-2-9111379 
pend on the efficient exploitation of light resource in the habitat by modification of vegetative crown development, as light is the most dominant limiting factor affecting plant growth (Ali and Kikuzawa 2005b, 2005c). To provide the crown a framework for effective display of photosynthetic organs, plants react to environmental conditions by morphologically changing and arranging modular units such as EU (Niinemets 2010). In view of optimal growth conditions for light interception, a significant relationship between shoot morphology and EU length and leaf size, demography and spacing is to be expected (Ishii and Asano 2010). Variation in above relationships could lead to a different crown shape, even under similar morphological rules of growth (Hiura 1998).

Aucuba japonica Thunb. var. japonica showed various changes in different levels of plant organization. The responses of phytoelements such as differences in EU length (Yagi and Kikuzawa 1999, Ishihara and Kikuzawa 2004) anisophylly varied according to habitat light conditions for the development of functional shoot morphology (Goebel 1900, Ali and Kikuzawa 2005c) and ultimately crown formation. Along with EU morphology, the distribution of EUs in the crown varies in accordance with habitat light conditions. Such variations exist even within a single crown (Ali and Kikuzawa 2005b).

With the likely change in the passage of time habitat light conditions, so do will the plant's response. To understand adaptation process of a plant, analyses of dynamic modifications of constituents of the crown on a long-term basis are essential (Niinemets 2010). In this paper, we examined whether: i) Aucuba japonica Thunb. var. japonica shows similar dynamic patterns of EU and leaf dimorphism in a spatio-temporal context; ii) change in structural and non-structural parts of the crown covary under different irradiation levels on a long-term basis; and iii) terminal buds have any role in crown development.

\section{MATERIALS AND METHODS}

Experimental material was Aucuba japonica Thunb. var. japonica belonging to the family Cornaceae. It is a dioecious, evergreen, woody understory shrub species native to the temperate zones of the Himalayas and Japan (Andersen et al. 1991). Its phyllotaxis decussates from vegetative to reproductive appendages (Hara 1980). Generally, flowering occurs at four years of age but it could be delayed upto 15 years (Kume and Ino 2000). Plant maturity is accompanied by anisophylly (Ali and Kikuzawa 2005c). Aucuba japonica typically grows under a closed forest canopy, but can be found in canopy gaps (Yamamura 1986).

At maturity, multiple EUs extend from a single reproductive bud, which leads to a sudden increase in leaf area in the crown posing severe potential self-shading in the crown (Takenaka et al. 1998, Ali and Kikuzawa 2005c). In the light limited understory conditions, development of efficient canopy to increase light acquisition (Tremmel and Bazzaz 1993) is key to survival. There is a particular dearth of holistic investigation on how developmental changes in phytoelements over years accrue into efficient canopy. Selection of the plant species Aucuba japonica helped to determine dimorphism of organs (e.g., leaves, EUs) of simultaneous origin. Moreover, decussate phyllotaxy allowed to identify organs of simultaneous origin.

We selected ten adult male plants of about 20-25 years of age and plant age was determined by counting the times of branching (Kume and Ino 2000). Each plant had a primary stem that branched on/above the ground and appeared multi-stemmed. All individuals were independent (visual observation) i.e., discrete type (Isobe and Kikuchi 1989).

Various morphological changes in the shoot system initiate in the terminal buds and accelerate further following the bud break. Thus, an understanding of the bud structure is essential for understanding branching pattern, anisophylly etc. and their roles in crown development. $\mathrm{Au}$ cuba produces two types of buds viz. terminal vegetative and terminal reproductive. The terminal buds commonly have two pairs of bud scales (Hara 1980) and each scale contains a small axillary bud primordium in its axil. Thus, a terminal reproductive bud has the potential to extend four EUs on the apex of the supporting EU. The axillary buds of the terminal reproductive bud develop into new anisphyllous EUs. The EUs subtended by the second/ distal pair of bud scales are generally more vigorous than those of the first/basal pair (Hara 1980, Ali and Kikuzawa 2005a). Despite simultaneous inception of axillary bud primordium (Hara 1980, Ali and Kikuzawa 2005a), the EUs subtended by a pair of bud scales do not grow equally. That is, the axillary buds of the terminal vegetative bud do not develop into EUs. Only the apical meristem of the terminal vegetative bud develops into a single new isophyllous EU. Juveniles (seedlings) and sprouts produce a terminal vegetative bud that extends into a single new EU (Kume and Ino 2000). In the mature crown, terminal vegetative buds are formed on the shorter EUs in the lower extremely shaded part and are smaller than the terminal reproductive buds (Ali and Kikuzawa 2005a). These EUs may begin to produce terminal reproductive buds again if 
their size exceeds the threshold size for flowering (Kume and Ino 2000).

To describe the features of dimorphism of different phytoelements and their integration in a functional crown development, we used various methods to measure the different but inter-related parameters. These methods and parameters are explained below.

\section{Extension unit (EU)}

"EU" was defined as the lateral axis that had grown out in a year from the terminal buds (Hara 1980) or sprouted on stems (Ali and Kikuzawa 2005b). This definition was applied to current-year or older EUs (in basipetal direction) with leaves.

\section{Measurement of phytoelements in different layers of crown}

To identify the layer-wise distribution of phytoelements, their morphological variations and growth dynamics quantitatively, we stratified a crown into horizontal layers of $50 \mathrm{~cm}$ depth each. We built vertical columns through the layers of a crown and such sampling column was replicated two to four times at different sites of a crown depending on its size. The randomly selected dimensions of a column on the horizontal plane were $60 \mathrm{~cm} \times 50 \mathrm{~cm}$. As a result, the dimensions of a measuring unit in a layer were as follows: $60 \mathrm{~cm} \times 50 \mathrm{~cm} \times 50 \mathrm{~cm}$ (vertical depth of a layer). Within each unit, we measured EU length, counted leaf numbers produced on the EU (fallen leaves were identified by leaf scar on EU), number of EUs per bud and number of dead EUs for the period of 1998-2001. In the case of trespassing boundary of a measuring unit by an EU, we sampled the EU only if nearly $80 \%$ of its length (visual observation) was inside the unit, or was otherwise excluded. However, such an event was infrequent.

\section{Measuring light}

We measured instantaneous light on each layer along the vertical column by putting sensors (LI-190SA; Li-Cor, Inc., Lincoln, NE, USA) at the center of the column as well as in the open. This measurement was carried out for all replications of the sampling column (two to four times depending on the individual crown size). The difference in photon flux density (PFD) between two consecutive sensors was regarded as light absorbance by the respective layer. Relative photon flux density (RPFD) was estimated in relation to PFD in the open. We measured light between 10:30 am and 2:00 pm standard time on an overcast day around the middle of August.

Additionally, we measured instantaneous light on the sample plants (five to ten locations per crown, depending on the individual crown size) using the same sensors to characterize light conditions on and around the sample plants. After this, RPFD was estimated following the same method described earlier. Irradiance level (on the sample plants) ranged from approximately $0.95 \%$ RPFD in closed sites to $8.5 \%$ RPFD in relatively open sites.

\section{Grouping of sample plants}

To compare within and between crowns, specifically the variation in phytoelements, we grouped individuals into two insolation regimes. We formed two groups comprising three individuals each. Four sample plants with intermediate insolation were kept aside to make the groups reasonably discrete. Group one (Gr. I) plants were considered as deeply shaded whose RPFD ranged from $0.95-1.4 \%$ while group two (Gr. II) plants were treated as only lightly shaded with varying light levels from $2.5-8.5 \%$ RPFD.

\section{Measurement of leaf size}

We measured laminar length and width ( 4,088 leaves) of fully expanded leaves on selected EUs during the period from 1999 to 2003 . Then leaf area was estimated by the equation, $\mathrm{Y}=0.682 \times \mathrm{X}(r=0.999)$ where, $\mathrm{X}=$ lamina length $\times$ width) (Ali and Kikuzawa 2005a).

\section{Estimation of distribution leaf area density in the crown}

Leaf area density $\left(\mathrm{m}^{2} / \mathrm{m}^{3}\right)$ was estimated as the product of mean leaf size and the number of leaves present in unit crown space.

\section{Clump}

We selected twenty EUs (grown in 1998) per individual in different layers covering the entire crown. On those selected EUs, measurement of the EU length, the number of EUs per bud, the number of leaves per EU, the leaf laminar dimensions and the number of dead EUs were determineduntil November 2003. The entire extension growth (from 1998 to 2003) of a selected EU in the crown was defined as a clump (Fig. 1). The clump signified two important ecological aspects of dynamics of leaf display 


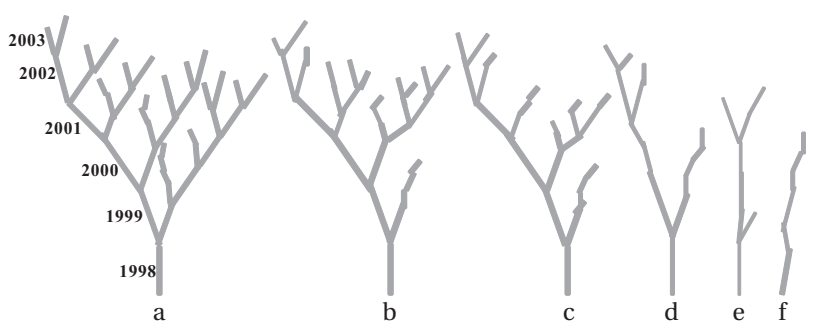

Fig. 1. Some observed branching patterns in Aucuba japonica Thunb. Clump forms of (a), (b), and (c) are generally found at brighter sites and in the uppermost layer of the crown. In these cases, the plant mostly produces terminal reproductive buds where multiple extension units (EUs) elongate from a single bud. Clump forms of (d), (e), and (f) are often found at darker sites and at the lower part of the crown where buds are mostly of the terminal vegetative type that can give birth to a single shorter EU. They can resume production of a terminal reproductive bud if their size exceeds the critical size (dry weight) for flowering.

structures and pace of space acquisition.

\section{Branching ratio}

Branching ratio was defined as the ratio of the number of current EUs to the number of one year old EUs. This was applied to compare the branching frequency in different layers of the crown and among the sample plants as well.

\section{Measurement of bud size}

We measured the bud length and diameter of 300 terminal reproductive buds on 300 hundred adult EUs of similar length ( 30 buds/plant; 15 buds at the apex layer and 15 buds at the lower most layer of the crown). Measurement was done in November (current season) and at the end of March next year before bud break. Buds were rather elliptical, wider in direction of the first pair of bud scales while suppressed in direction of the second pair. Thus, the bud diameter was measured in two directions perpendicular to each other (a, b \& a > b) and then averaged. Assuming a conical shape, we estimated the bud size (volume) by the equation, $\mathrm{S}=\frac{1}{3} \pi \mathrm{r}^{2} \mathrm{~h}$, where, $\mathrm{r}=$ radius [estimated as $\mathrm{r}=\mathrm{D} / 2$ while, $\mathrm{D}=(\mathrm{a}+\mathrm{b}) / 2]$ and $\mathrm{h}=$ bud length .

\section{Measurement of the degree of EU dimorphism (DD)}

EU is the prime structural unit for leaf display in which the length of EU matters. Length difference among sister EUs (originating from a single bud) initiates in the bud and the difference was furthered followed by bud break. We expressed DD as the ratio of the longer $\mathrm{EU}\left(\mathrm{L}_{\mathrm{EU}}\right)$ to the shorter EU $\left(\mathrm{S}_{\mathrm{EU}}\right)$ extending from a single bud. Intermedi- ate $\mathrm{EU}(\mathrm{s})$ were excluded in determining $\mathrm{DD}$ in which the number of sister EUs exceeded two.

\section{Measurement of the degree of anisophylly}

We expressed the degree of anisophylly as the ratio of the leaf area of two leaves opposite to each other on a node. For the anisophyllous nodes, we used the ratio of outer to inner leaf area. The leaf positions were designated in regard to the position of the inflorescence where the outer leaf was positioned away from the inflorescence and the inner was positioned on the side of the inflorescence. The outer leaf was always larger than the inner leaf.

\section{Statistical analyses}

For statistical analyses, Two-way ANOVA, Mann-Whitney U test, and correlation analysis were used wherever applicable, and for obtaining graphs, SPSS Inc. (Chicago, IL, USA) and Excel were used.

\section{RESULTS}

The leaf area of plants was mostly displayed in the upper peripheral layer of the crown and decreased towards the lower layers. Both groups of plants showed similar patterns of leaf area display within the crown. For any comparable layers of the crown, Gr. II plants displayed leaf area density (LAD) twice to that of Gr. I. For Gr. I, there was a significant difference $(P<0.001$, One-way ANOVA) among the layers of crown in terms of LAD. Also, LAD of the two consecutive layers was significantly different $(P$ $<0.001$, Mann-Whitney U test) except for the two lower most layers. Similarly, for Gr. II, there was a significant difference $(P<0.05$, One-way ANOVA) among layers of crown but difference was insignificant between two consecutive layers except in the apex and the lowest layer $(P$ $<0.001$, Mann-Whitney U test). Also, there was a significant difference $(P<0.001$, Mann-Whitney U test) between the two lower most consecutive layers (Fig. 2a). LAD was significantly higher $(P<0.05$, One-way ANOVA) for Gr. II than Gr. I. The apex layer of the crown mostly absorbed incident light and this absorption was significantly higher $(P<0.001$, Two-way ANOVA) for Gr. II than Gr. I. Correlation between $\mathrm{LAD}$ and light adsorption was insignificant $(P>0.5, r=-0.077)$ for Gr. I while a significantly positive correlation ( $P<0.05, r=0.52)$ was found for Gr. II (Fig. 2b).

A significant positive correlation between incident light and branching ratio (Fig. 2c) indicated that branch- 
ing ratio was a function of light. The branching ratio was significantly higher in plants (Gr. II) under brighter conditions. Branching ratio decreased from the top towards the bottom of the crown and the difference was significant between the top and the lowest layer of the crown (Fig. 2d). Lower branching ratio led to the development
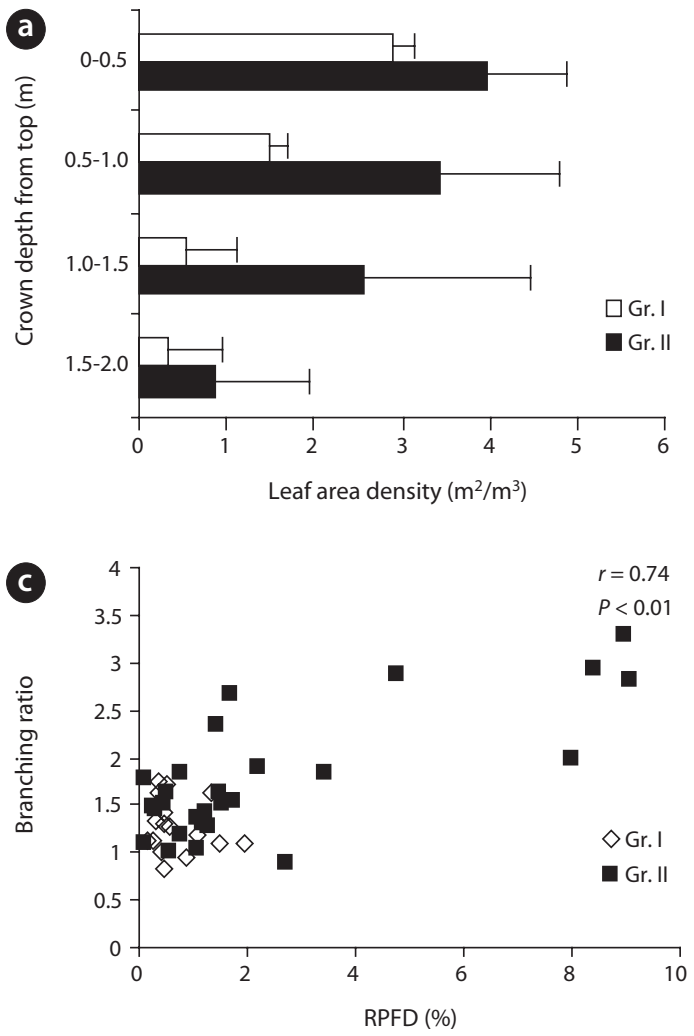

of a sparse crown while the reverse was true for higher branching ratio. The mean number of EUs and the mean extension growth per clump were closer to each other at earlier age of the plants but differed subsequently over time (Fig. 3a and 3b). Incremental mean EU growth $(\mathrm{cm})$ per clump over time indicated expansion of the crown at
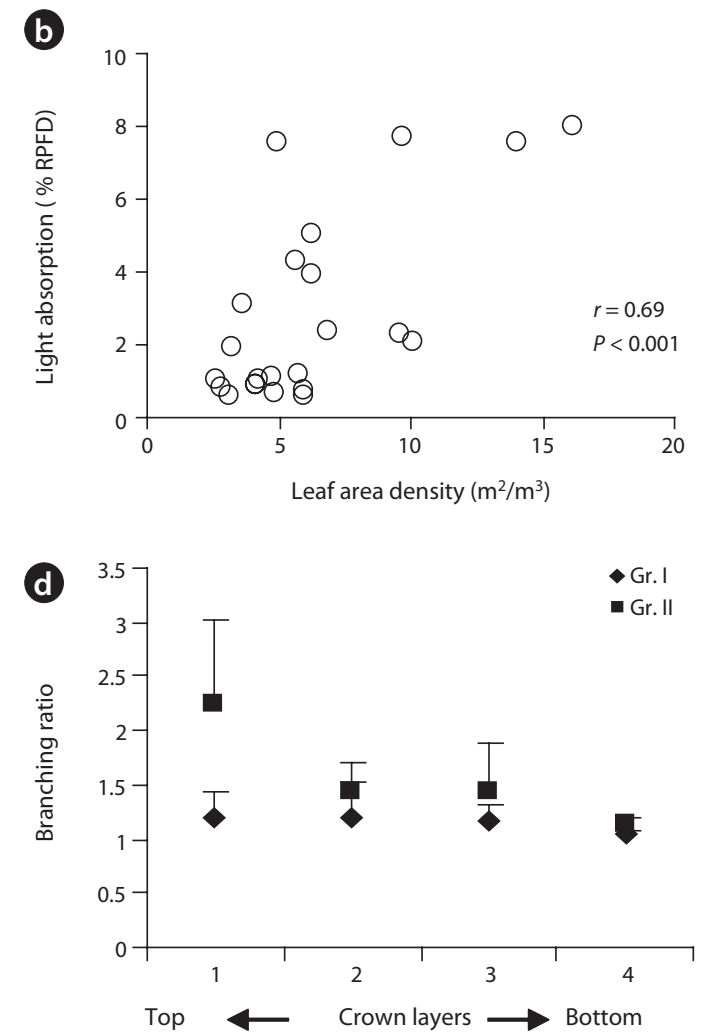

Fig. 2. The absorption of incident light by different layers of crown and its impact on branching. (a) Leaf area distribution varied in different layers of a crown. For both groups of plants, the leaf area in the crown was displayed mostly in the apex layer and decreased towards the bottom layers. (b) Incident light on the crown was absorbed mostly by the apex layer of the crown and absorption was higher for group (Gr.) II than for Gr. I. (c) A correlation exists between incident light and branching ratio data was pooled from all the layers for all replications of all the ten individual plants. (d) The branching ratio at different layers of a crown the branching ratio was significantly higher $(P<0.001$, Two-way ANOVA) for Gr. II than for Gr. I. There was an insignificant $(P>0.05)$ interaction effect between plant groups, with crown layers indicating the effect of light on branching. Data was pooled from all the replications of individual plants of the groups; the error bars represent standard deviation. RPFD, relative photon flux density.
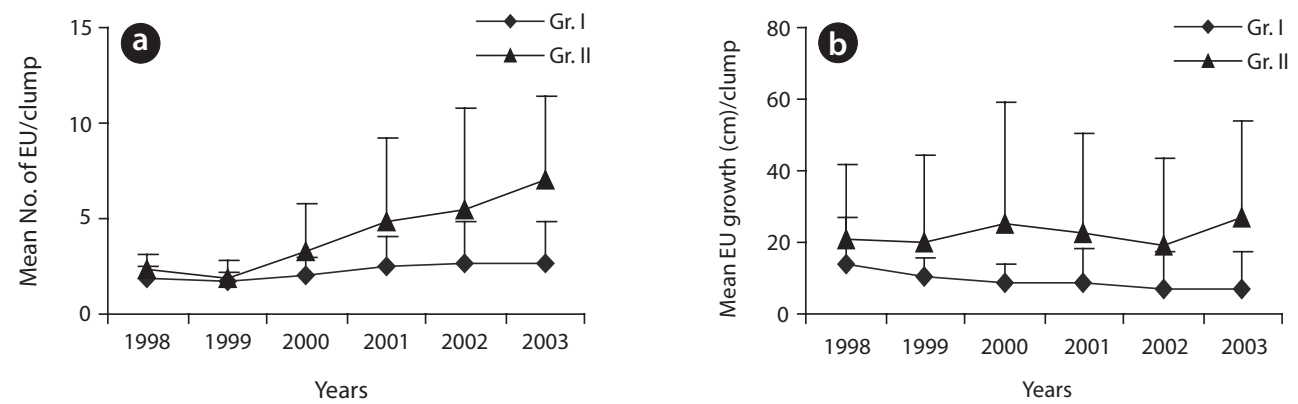

Fig. 3. Spatio-temporal variation in annual extension growth. (a) The mean number of extension units (EUs) per clump in different years. (b) The mean total of EU growth $(\mathrm{cm})$ per clump per annum. Data was pooled from all the individuals of group (Gr.) I and of Gr. Il; the error bars represent standard deviation. 

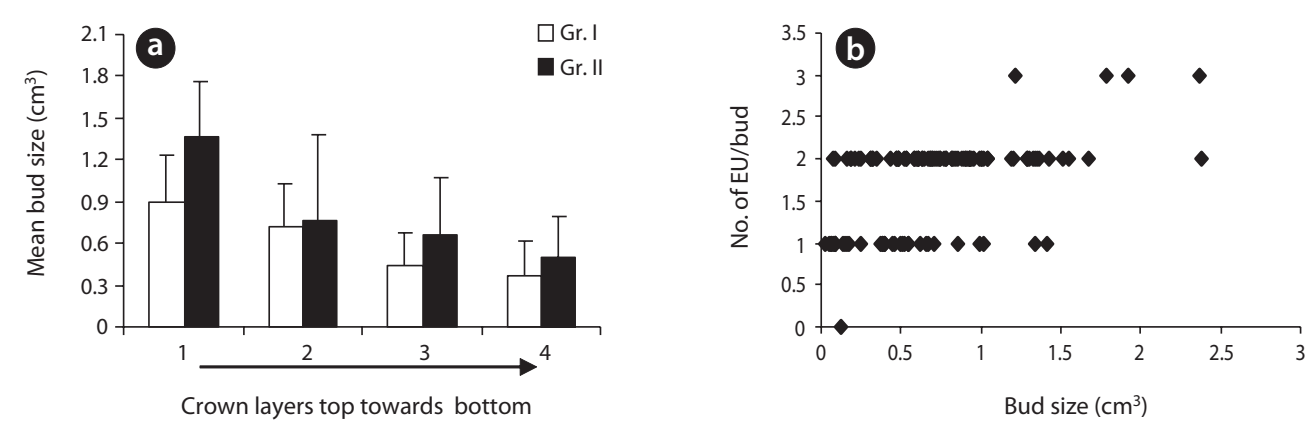

Fig. 4. Bud size and its relation to branching. (a) The difference in bud size between the groups of plants—-the bud size was significantly larger for group (Gr.) II than for Gr. I $(P<0.01$, Two-way ANOVA). There was an insignificant $(P>0.5)$ interaction effect between the plant groups; and crown layers indicated the effect of light on bud size. (b) There was a correlation between bud size and number of EUs per bud $(r=0.51, P<0.01)$. EUs developed from the axillary bud in the axil of the bud scale of the terminal reproductive buds and a terminal reproductive bud has the potential to give birth to zero to four EUs per annum.

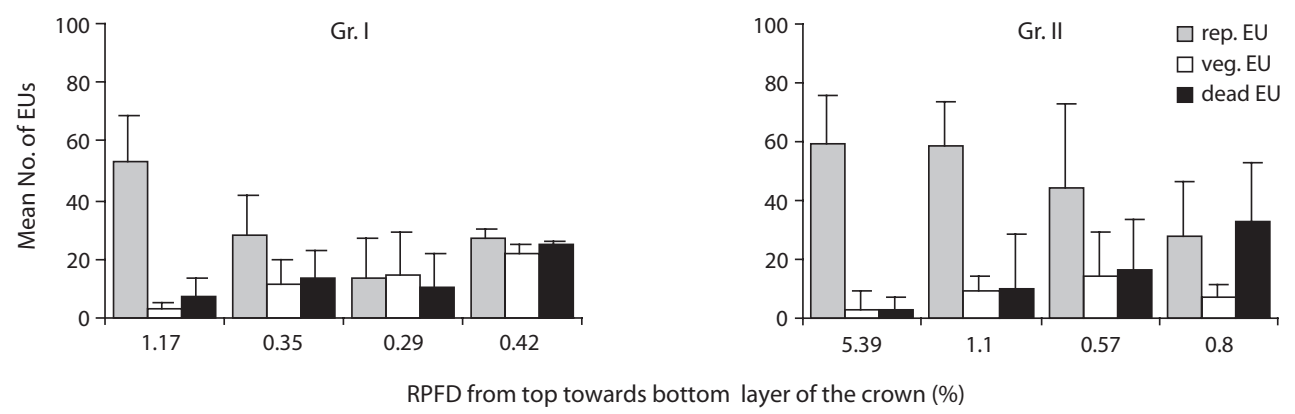

Fig. 5. The frequency distribution of the extension unit (EU) population within a crown. Both groups showed similar patterns of EU distribution in the crown though there was a quantitative difference in EU distribution between groups as well as among different layers of the crown. rep. EU, EU extended from a reproductive bud; veg. EU, EU extended from a vegetative bud; dead EU, an EU which was dead at the time of measurement.

a higher rate at brighter sites and diminishing mean EU growth $(\mathrm{cm})$ indicated expansion of the crown at a lower rate at darker sites. Annual structural growth, the sum of EUs, was significantly higher $(P<0.05$ for 1998; $P<0.01$ for the rest of the years, One-way ANOVA) for Gr. II than Gr. I (Fig. 3b).

Plants (Gr. II) at brighter sites produced significantly larger buds $(P<0.05$, One-way ANOVA) than plants at darker sites. Within a single crown, bud size was significantly larger in the top layer than in the lower most layer $(P<0.001$, Mann Whitney U test) while in a few cases, insignificant difference in bud size between two consecutive layers was found (Fig. 4a). A significant positive correlation $(r=0.51, P<0.01)$ between bud size and number of EUs per bud (Fig. 4b) indicated the larger the bud size the higher the branching ratio. However, this was not applicable to terminal vegetative buds.

In the top layer of the crown, nearly $60 \%$ of EUs were produced from terminal reproductive buds and the proportion gradually decreased down the crown. The proportion of EUs produced from vegetative buds increased towards the lower layers of the crown. Both groups showed a similar pattern of EU production throughout the crown. Gr. I had a greater proportion of EUs produced from vegetative buds compared to Gr. II. EU death showed an increasing trend towards the bottom of the crown. Gr. II had greater EU mortality in the lowest layer than Gr. I (Fig. 5).

Plants at brighter sites produced significantly larger mean leaf size throughout the period of 1999-2003 (Twoway ANOVA). The mean leaf size gradually decreased over time for both Gr. I and Gr. II as the plants aged (Table 1). For both groups, mean leaf size was significantly different between the top and the lowest layer of the crown. Leaf size difference within the crown was significantly higher for Gr. II than for Gr. I (Table 2).

For both groups, there was a positive correlation $(P<$ 0.01 ) between EU length and number of leaves per EU throughout the crown of sample plants. EU length decreased significantly $(P<0.05$, Mann-Whitney $\mathrm{U}$ test $)$ from top towards the bottom layer of the crown and the number of leaves per EU followed a similar declining pattern (Fig. 6). Generally, the EU length and number of leaves per EU decreased significantly $(P<0.001$, One-way ANOVA) from the beginning (1998) to the end (2003) of 

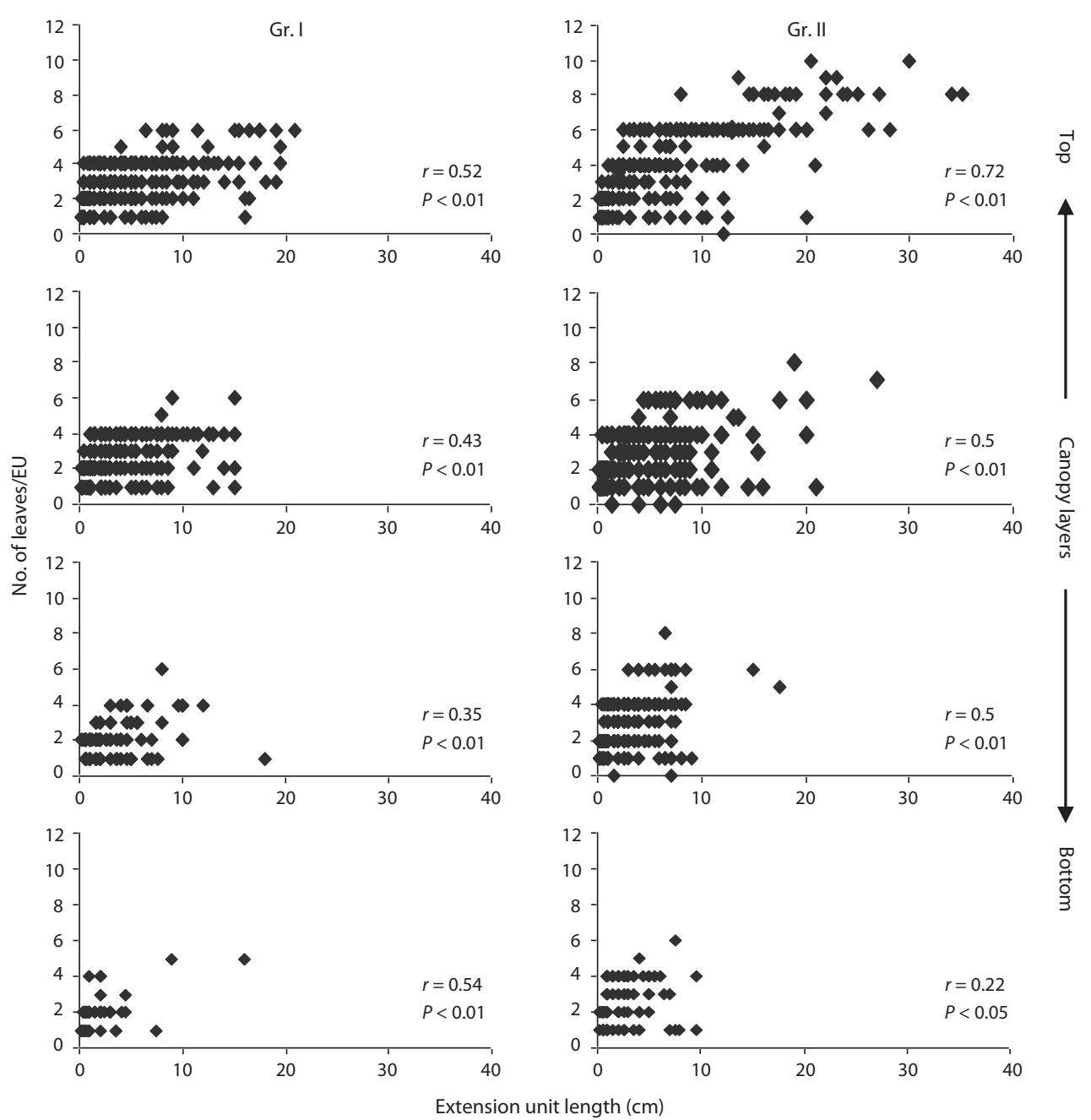

Fig. 6. The relationship between the extension unit (EU) length and the number of leaves on an EU-both groups of plants showed similar distribution patterns of EU and leaves in different layers of the crown. EUs and leaves were mostly distributed in the apex layer of the crown while the lower layers were degraded. Note: sometimes epicormic EUs in the lower crown could be longer with a larger number of leaves.

Table 1. Differential variation in leaf size $\left(\mathrm{cm}^{2}\right)$ between the groups of plants in different years

\begin{tabular}{|c|c|c|c|c|}
\hline \multirow{2}{*}{ Year } & \multicolumn{3}{|c|}{ Mean leaf size $( \pm$ SE) } & \multirow{2}{*}{$\begin{array}{l}\text { Level of significance } \\
\text { Between Gr. I \& Gr. II }\end{array}$} \\
\hline & Gr. I & Intermediate Gr. & Gr. II & \\
\hline 1999 & $37.15 \pm 1.09$ & $36.25 \pm 1.12$ & $42.43 \pm 1.17$ & $P<0.001$ (Two-way ANOVA) \\
\hline 2000 & $31.37 \pm 1.04$ & $31.28 \pm 0.94$ & $37.51 \pm 1.11$ & \\
\hline 2001 & $28.22 \pm 1.04$ & $29.36 \pm 1.12$ & $31.73 \pm 1.04$ & \\
\hline 2002 & $27.74 \pm 0.91$ & $28.50 \pm 0.73$ & $30.08 \pm 0.73$ & \\
\hline 2003 & $26.83 \pm 1.05$ & $26.76 \pm 1.13$ & $30.09 \pm 0.82$ & \\
\hline
\end{tabular}

Insignificant $(P>0.05)$ interaction effect indicated that the gradual decrease in leaf size was mainly due to the age effect of plants.

Table 2. Within crown leaf size $\left(\mathrm{cm}^{2}\right)$ variation (data pooled from 1999-2003 for each group)

\begin{tabular}{cccc}
\hline \multirow{2}{*}{ Plant groups } & \multicolumn{2}{c}{ Mean leaf size $( \pm$ SE) } & Level of significance \\
\cline { 2 - 3 } & Top layer & Bottom layer & \\
\hline Group I & $31.91 \pm 0.71$ & $28.83 \pm 0.74$ & $P$ 0.001 (Two-way ANOVA) \\
Group II & $37.05 \pm 0.64$ & $29.21 \pm 0.59$ & \\
\hline
\end{tabular}

Significant $(P<0.01)$ interaction effect signified additive impact of temporal variation in leaf size. 
the study. During the study period, the EU length and number of leaves per EU decreased significantly $(P<0.05$, Mann-Whitney $U$ test) between two consecutive years (Fig. 7).

The phenomenon of EU dimorphism is accompanied by reproductive maturity and is applicable to EUs extend- ed from reproductive buds in the mature crown. Aucuba japonica was shown to express EU dimorphism irrespective of habitat light conditions throughout the crown. The DD was significantly higher $(P<0.001$, One-way ANOVA) in Gr. I than in Gr. II. DD gradually increased as the plants kept growing and became aged. The mean DD
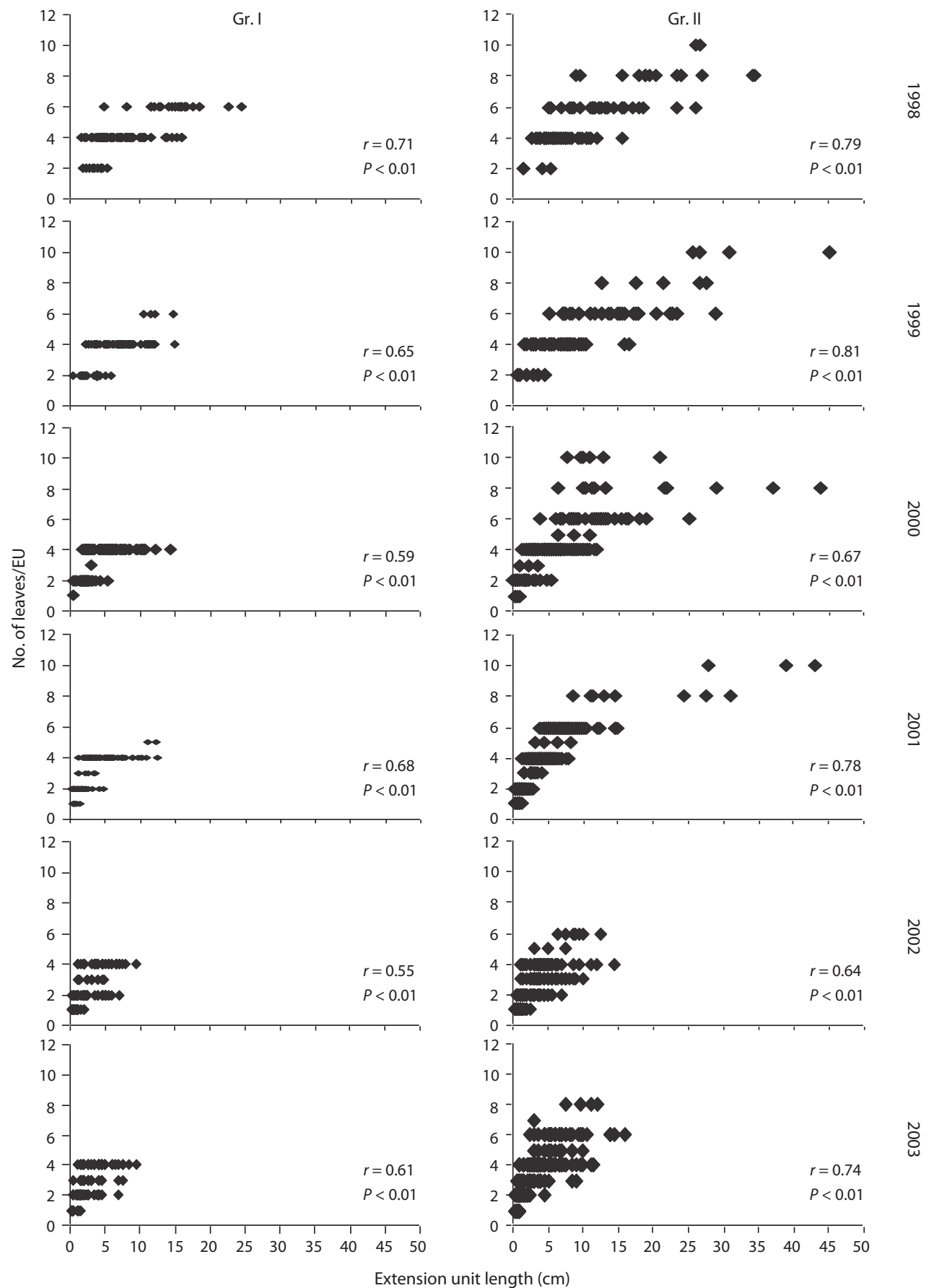

Fig. 7. The relationship between the extension unit (EU) length and the number of leaves on an EU over the years-both groups of plants showed a similarly decreasing pattern of EU length and the number of leaves per EU. Regression analyses showed a significant relationship between the EU length and the number of leaves on EU $(P<0.05)$. 
increased significantly $(P<0.001$, Mann Whitney U test $)$ during the period from 1998 to 2003. However, the mean DD did not differ significantly $(P>0.05$, Mann-Whitney $\mathrm{U}$ test) between two consecutive years (Fig. 8). Within the crown, plants expressed a significantly higher degree of anisophylly in the lower part than in the upper part (Fig.

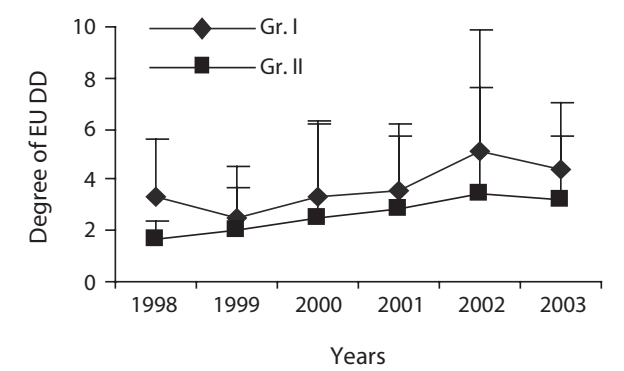

Fig. 8. The temporal variation in the degree of extension unit (EU) dimorphism (DD), the ratio of long $\mathrm{EU}\left(\mathrm{L}_{\mathrm{EU}}\right)$ to short $\mathrm{EU}\left(\mathrm{S}_{\mathrm{EU}}\right)$ extending from a single terminal reproductive bud. Plants under shaded conditions expressed significantly $(P<0.001$, Two-way ANOVA) greater DD. Both groups showed an overall gradual increase in DD as the plants aged. The interaction effect between plant groups and years was insignificant $(P>0.3)$ indicating the effect of bud size (owing to physical resources) on DD. Data was pooled group wise; error bars represent standard deviation.
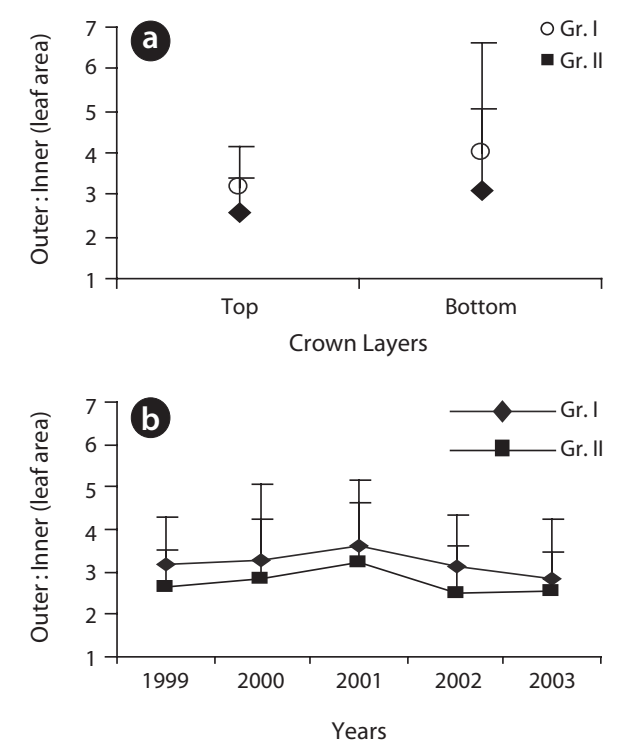

Fig. 9. The spatio-temporal variation in anisophylly. (a) Within crown variation in the degree of anisophylly - both groups showed similar patterns in which the degree of anisophylly was significantly higher in the bottom layer than in the apex layer $(P<0.001$, Two-way ANOVA). (b) Plants under darker conditions expressed a significantly higher $(P<0.001$, Two-way ANOVA) anisophylly than plants in brighter sites over time. The interaction effect between plant groups and years was insignificant $(P>0.5)$ which indicated the effect of bud size, owing to physical resources on the degree of anisophylly. Data was pooled group wise. Leaves produced in 1998 had mostly fallen at the time of the first measurement in 2001; error bars represent standard deviation. 9a). The degree of anisophylly was significantly higher at darker sites over the years though year-to-year variation was observed (Fig. 9b).

\section{DISCUSSION}

Structural variations in EUs and their spatial distribution determine foliage distribution in the crown (Ali and Kikuzawa 2005b) and ultimately plant production (Osada et al. 2002, Ishii and Asano 2010) by reducing potential self-shading. Terminal buds, the breeding house of EUs, play a key role in crown formation. At brighter sites and at the top of the crown, terminal buds were mostly reproductive and larger in size leading to a higher branching ratio. They also produced many longer EUs. As a result, extension growth per clump per year (Fig. 3a and $3 \mathrm{~b}$ ) was higher at brighter sites than at darker sites. A higher branching ratio along with larger extension growth provided ample structural support for larger leaf area both in terms of leaf number and individual leaf size (Ali and Kikuzawa 2005b). Due to the positive correlation between leaf area and structural support (Osada et al. 2002), leaf production mostly concentrated in the apex peripheral layer of the crown. Both groups of plants showed a similar pattern of leaf production within a crown. Under darker conditions, plant crowns expanded at a diminishing rate to maintain rather a stable stature. At darker sites, even within a single crown, a significantly lower branching ratio (due to smaller bud size) led to the development of a sparse crown. Contrarily, with a significantly higher branching ratio together with many longer EUs on plants at brighter sites, crowns became denser and expanded at a progressive rate. A higher branching ratio which led to increased EU density especially in the peripheral layer of the crown affected light transmittance through the crown (Ali and Kikuzawa 2005b). However, the gradual decrease in mean leaf size over the years helps plants to minimize self-shading (Osada et al. 2002). Incremental mean EU growth per clump over time indicates expansion of the crown at a higher rate at brighter sites and diminishing mean EU growth indicates expansion of the crown at a lower rate at darker sites (Fig. 3b).

EU dimorphism which helps to reduce self-shading (Yagi and Kikuzawa 1999) initiates in the bud and the extent of dimorphism depends on the size of the terminal bud (Ali and Kikuzawa 2005a). The DD is further amplified following bud break. A significant difference in the DD between the groups indicates the influence of light level on amplification of dimorphism. Increased EU 
dimorphism (due to differential height growth) i.e., increased difference in length between longer and shorter EUs extended from a single bud enabled leaf display at different heights. Also, it might lead to having openings in the peripheral layer having an enhanced light penetration to leaves on shorter EUs as well as to older leaves in the lower layers, which remain photosynthetically active for a longer time. Greater dimorphism leads a plant to reap more benefit under light deficient conditions where selfshading is critical for survival (Yamada et al. 2000).

Having bi-directional functions (space acquisition and the utilization of acquired space around the base of a longer EU), EU dimorphism has increased the efficiency of the plants (Yagi and Kikuzawa 1999), at least, for the current season. EU dimorphism simultaneously offers discriminate competition between the leader EU and the shorter EU which in turn particularly affects the survival of shorter EUs. With the progress of time and the emergence of new EUs above, older EUs, especially the shorter EUs, moved downward relatively faster and sank deeper in the expanding crown under brighter conditions. At the same time, the photosynthetic capacity of EUs declined depending on the rate and temporal context of the progression from the exterior to interior crown position that varies with the rate of EU growth and leaf turnover (Kikuzawa et al. 2009). In that case, shorter EUs may no longer remain a source of carbon and rather turn into a sink. The smaller EUs invest a significantly greater proportion of their dry mass into leaves and they become smaller with ramifications each succeeding year. As a result, the growth of smaller EUs was suspended and died (Kume and Ino 2000). Subsequently, the role of shorter EUs reduced in terms of photosynthetic gain and crown construction in the long run (Seiwa et al. 2006).

At darker sites or even in the lower layer of a mature crown, many smaller EUs, those which reached below the threshold level for flowering produced a terminal vegetative bud that extended a single smaller EU on the supporting axis (Kume and Ino 2000). Resource constraint prohibited flowering (Ishihara and Kikuzawa 2009) and thus, branching and crown development as well. If the EU size exceeds the threshold level, then flowering/branching could be resumed (Kume and Ino 2000). However, those smaller EUs are often suppressed by other larger EUs (Kume and Ino 2000) that cannot keep pace with crown expansion and die eventually. A denser crown under brighter conditions might cause greater heterogeneity of irradiance levels between the layers of a crown that lead to higher EU mortality due to correlative growth inhibition (Stoll and Schmid 1998, Takenaka 2000). This result caused in turn a higher degradation of the lower part of the crown at brighter sites than at darker sites. The deployment of fewer shorter EUs with fewer smaller leaves in the lower part of the crown together with the higher mortality of EUs accelerated resource translocation to the upper crown to maximize benefit (Ali and Kikuzawa 2005c). Thus, the denser apex layer of the crown developed at the cost of degradation of the lower part of the crown that led to internal recycling of nutrients. Such an internal recycling of nutrients was higher at brighter sites than at darker sites.

The variation in the degree of anisophylly (within and between crowns) was owing to the variation in bud size (Ali and Kikuzawa 2005a). Similarly, the changes in the degree of anisophylly over the years were due to bud size and these bud size variations could be explained, partially, by weather conditions and resource availability over time and space. However, the significance of greater anisophylly under shaded conditions lay in the essence of reducing self-shading since light was the single most limiting factor to plant life (Muraoka et al. 2003), and low levels of self-shading can have a large impact on net photosynthesis near the compensation-point light level (Givnish 1984). The problem of potential self-shading in the crown was resolved through the synchronization of increased EU dimorphism and anisophylly, and a gradual decrease in EU length and by making leaf size smaller over time. The decrease in mean EU length and individual leaf size and number of leaves per EU over the years well indicated the convergence of structural support and the leaf area towards efficient and functional crown development (Ishii and Asano 2010).

\section{CONCLUSION}

The morphology of phytoelements changed in a manner responsive to habitat light conditions and responses varied in a spatio-temporal context. There was significant integration of EU dimorphism, anisophylly, EU extension growth and leaf size variations in the crown to reduce potential self-shading. Shorter EUs had a mere role in crown construction but their death could cause the formation of gaps that were previously filled by them. EU dimorphism that caused discriminating competition among EUs, led to higher EU mortality and paved the way for resource translocation from darker to brighter sites of the crown. Terminal buds appeared as a key initiator of various changes in the phytoelements, which were amplified following bud break while bud types and their sizes acted as 
the function of light.

Long-term analyses of crown development revealed two different modes of crown expansion. At brighter sites, plant crown expansion was progressive while at darker sites, the crown expanded in a regressive manner and maintained a stable size. A plant's "diminishing growth phase" appeared earlier at shaded sites than at brighter sites.

\section{ACKNOWLEDGMENTS}

We express gratitude to the Botanical Garden Authority of Kyoto University for permission to conduct the experiments of this study. We would like to thank our colleagues at the Laboratory of Forest Biology, Kyoto University for their kind and continuous support during the experimental work. We are greatly indebted to Andrew Eagle, Macquarie University, Australia for reviewing the manuscript.

\section{LITERATURE CITED}

Ali MS, Kikuzawa K. 2005a. Anisophylly in Aucuba japonica (Cornaceae): an outcome of spatial crowding in the bud. Can J Bot 83: 143-154.

Ali MS, Kikuzawa K. 2005b. Plasticity in leaf-area density within the crown of Aucuba japonica growing under different light levels. J Plant Res 118: 307-316.

Ali MS, Kikuzawa K. 2005c. Shoot morphology of Aucuba japonica incurred by anisophylly: ecological implications. J Plant Res 118: 329-338.

Andersen PC, Knox GW, Norcini JG. 1991. Light intensity influences growth and leaf physiology of Aucuba japonica 'Variegata'. Hortscience 26: 1485-1488.

Givnish TJ. 1984. Leaf and canopy adaptations in tropical forests. In: Physiological Ecology of Plants of the Wet Tropics (Medina E, Mooney HA, Vazquez-Yanes C, eds). W. Junk, The Hague, pp 51-84.

Goebel K. 1900. Organography of Plants. Oxford University Press, Oxford.

Hallé F, Oldeman RAA, Tomlinson PB. 1978. Tropical Trees and Forests: An Architectural Analysis. Springer-Verlag, Berlin.

Hara N. 1980. Shoot development of Aucuba japonica I. Morphological study. Bot Mag Tokyo 93: 101-116.

Hatakeyama I, Murata G, Tabata H. 1973. A list of plants in the botanical garden of Kyoto University and some ecological data. Mem Fac Sci Kyoto Univ Biol 6: 91-148.

Hiura T. 1998. Shoot dynamics and architecture of sapling in Fagus crenata across its geographical range. Tress 12: 274-280.

Ishihara M, Kikuzawa K. 2004. Species-specific variation in shoot production patterns of five birch species with respect to vegetative and reproductive shoots. Can J Bot 82: 1393-1401.

Ishihara MI, Kikuzawa K. 2009. Annual and spatial variation in shoot demography associated with masting in Betula grossa: comparison between mature trees and saplings. Ann Bot 104: 1195-1205.

Ishii H, Asano S. 2010. The role of crown architecture, leaf phenology and photosynthetic activity in promoting complementary use of light among coexisting species in temperate forests. Ecol Res 25: 715-722.

Isobe H, Kikuchi T. 1989. Differences in shoot form and age of Aucuba japonica Thunb. corresponding to the microlandforms on a hill slope. Ecol Rev 21: 277-281.

Jones M, Harper JL. 1987. The influence of neighbours on the growth of trees. II. The fate of buds on long and short shoots in Betula pendula. Proc R Soc Lond B 232: 19-33.

Kikuzawa K, Yagi M, Ohto Y, Umeki K, Lechowicz MJ. 2009. Canopy ergodicity: can a single leaf represent an entire plant canopy? Plant Ecol 202: 309-323.

Kume A, Ino Y. 2000. Differences in shoot size and allometry between two evergreen broad-leaved shrubs, Aucuba japonica varieties in two contrasting snowfall habitats. J Plant Res 113: 353-363.

Maillette L. 1982. Structural dynamics of silver birch. I. The fates of buds. J Appl Ecol 19: 203-218.

Muraoka H, Koizumi H, Pearcy RW. 2003. Leaf display and photosynthesis of tree seedlings in a cool-temperate deciduous broadleaf forest understorey. Oecologia 135: 500-509.

Niinemets Ü. 2010. A review of light interception in plant stands from leaf to canopy in different plant functional types and in species with varying shade tolerance. Ecol Res 25: 693-714.

Osada N, Takeda H, Furukawa A, Awang M. 2002. Changes in shoot allometry with increasing tree height in a tropical canopy species, Elateriospermum tapos. Tree Physiol 22: 625-632.

Seiwa K, Kikuzawa K, Kadowaki T, Akasaka S, Ueno N. 2006. Shoot life span in relation to successional status in deciduous broad-leaved tree species in a temperate forest. New Phytol 169: 537-548.

Stoll P, Schmid B. 1998. Plant foraging and dynamic competition between branches of Pinus sylvestris in contrasting light environments. J Ecol 86: 934-945.

Takenaka A. 2000. Shoot growth responses to light microenvironment and correlative inhibition in tree seedlings 
under a forest canopy. Tree Physiol 20: 987-991.

Takenaka A, Inui Y, Osawa A. 1998. Measurement of threedimensional structure of plants with a simple device and estimation of light capture of individual leaves. Funct Ecol 12: 159-165.

Tremmel DC, Bazzaz FA. 1993. How neighbor canopy architecture affects target plant performance. Ecology 74 : 2114-2124.

van Volkenburgh E. 1999. Leaf expansion: an integrating plant behaviour. Plant Cell Environ 22: 1463-1473.

Whitney GG. 1976. The bifurcation ratio as an indicator of adaptive strategy in woody plant species. Bull Torrey Bot Club 103: 67-72.

Yagi T, Kikuzawa K. 1999. Patterns in size-related variations in current-year shoot structure in eight deciduous tree species. J Plant Res 112: 343-352.

Yamada T, Okuda T, Abdullah M, Awang M, Furukawa A. 2000. The leaf development process and its significance for reducing self-shading of a tropical pioneer tree species. Oecologia 125: 476-482.

Yamamura Y. 1986. Matter-economical roles of the evergreen foliage of Aucuba japonica, an understory shrub in the warm-temperate region of Japan. 1. Leaf demography, productivity and dry matter economy. Bot Mag Tokyo 99: 323-332.

Yano S, Terashima I. 2004. Developmental process of sun and shade leaves in Chenopodium album L. Plant Cell Environ 27: 781-793. 